\title{
Pulse Wave Velocity in Lower-Limb Arteries among Diabetic Patients with Peripheral Arterial Disease
}

\author{
Hisayo Yokoyama, Tetsuo Shoji, Eiji Kimoto, Kayo Shinohara, Shinji Tanaka, \\ Hidenori Koyama, Masanori Emoto, and Yoshiki Nishizawa
}

Department of Metabolism, Endocrinology and Molecular Medicine, Osaka City University Graduate School of Medicine, Osaka, Japan.

\begin{abstract}
Objective: Patients with type 2 diabetes mellitus are at an increased risk of atherosclerosis including peripheral arterial disease (PAD). The purpose of this study was to examine the possible alteration in pulse wave velocity (PWV) in lower-limb arteries among diabetic patients with PAD.

Methods: We measured brachial-ankle PWV (baPWV) using an automatic device in 101 healthy control subjects and 102 type 2 diabetic patients including those with PAD.

Results: Diabetic patients without PAD showed a higher baPWV than the healthy control subjects. There was no significant difference in baPWV between the right and left legs in these groups. In contrast, among diabetic patients with PAD, baPWV was significantly lower in the affected legs than in the non-affected legs, and the reduction in baPWV was greater in those with lower ankle-brachial pressure index (ABI). In the patients with PAD who received percutaneous transluminal angioplasty, both baPWV and $A B I$ were increased following successful vessel dilatation.

Conclusions: These results suggest that baPWV is increased in diabetic patients, whereas it is decreased in the affected legs in diabetic patients with PAD. Widening of the right-left difference in baPWV may be a novel marker of PAD. J Atheroscler Thromb, 2003; 10: 253-258.
\end{abstract}

Key words: Type 2 diabetes, Ankle-brachial pressure index, Percutaneous transluminal angioplasty, The absolute difference between right-left

\section{Introduction}

Peripheral artery disease (PAD) is one of the major manifestations of atherosclerosis, and the prevalence of PAD is higher in patients with diabetes mellitus than in the general population (1). Presence of PAD is a predictor of amputation (2) and poor survival (3) in type 2 diabetic patients.

Atherosclerosis has two key components, namely thick-

Address for correspondence: Tetsuo Shoji, Department of Metabolism, Endocrinology and Molecular Medicine, Osaka city University Graduate School of Medicine, 1-4-3, Asahi-machi, Abenoku, Osaka, Osaka 545-8585, Japan

E-mail: t-shoji@med.osaka-cu.ac.jp

Received January 31, 2003.

Accepted for publication April 9, 2003. ening (atherosis) and stiffening (sclerosis) of arterial wall (4). Arterial stiffness can be assessed by measuring physiological properties of arteries including pulse wave velocity (PWV), stiffness index $\beta$, compliance and others. Due to recent important observations, arterial stiffness has gained much attention. Aortic PWV is associated with left ventricular hypertrophy (5), increased pulse pressure (6), and the risk of cardiovascular mortality (7-9). In addition, our recent study (10) showed that stiffness index $\beta$ of femoral artery was closely associated with symptoms of lower limb PAD independent of the thickness of the femoral artery in those with type 2 diabetes mellitus. Another recent study by Suzuki et al. (11) demonstrated that brachial-ankle PWV (baPWV) correlated inversely with blood flow at the popliteal artery in patients with type 2 diabetes mellitus, but they excluded those with 
PAD. These studies suggest the importance of lowerlimb arterial stiffness in impaired peripheral circulation.

There is only a limited amount of information regarding possible changes in PWV among patients with PAD. In an early study by Haynes (12), no significant difference was observed in PWV of the aorta, carotid-brachial, or femoral-dorsalis segments between subjects with and without PAD. Safar et al. (13) measured brachial-radial PWV in 20 patients with PAD, and found that it remained within the normal range. In contrast, Simonson et al. (14) and Eliakim et al. (15) reported that heart-feet PWV, and femoral-dorsalis PWV were reduced in patients with PAD, respectively. Thus, the results are inconsistent, and none of these studies evaluated the reversibility of PWV of lower-limb arteries following dilatation of stenosed arteries. In addition, no study evaluated PWV in the lower extremities in diabetic patients with PAD, a population with an extremely elevated risk for amputation and mortality.

In the present study, we measured baPWV in type 2 diabetic patients with and without PAD and healthy control subjects to investigate the effects of diabetes and PAD to PWV on lower-limb arteries.

\section{Subjects}

The subjects were 102 patients with type 2 diabetes mellitus and 101 healthy subjects who gave informed consent to participate in the study (Table 1).

The diabetic patients were consecutive patients admitted to Osaka City University Hospital to attend an educational course on diabetes, for the treatment of diabetes, or for the evaluation and treatment of PAD. The diagnosis of diabetes mellitus was based on the criteria of the American Diabetes Association (16). No subject had a history of ketoacidosis or positivity for anti-GAD antibody. Among the patients, 15 were treated with insulin injection for control, 56 with oral anti-diabetic agents, and 31 with lifestyle modification alone. In addition, 36 received medication for dyslipidemia and 35 for hypertension.

Presence of PAD was diagnosed when the subjects had both PAD symptoms and a reduced ankle-brachial pressure index $(A B I)$ less than 0.9 . History was carefully taken for the leg symptoms attributable to PAD. Intermittent claudication was taken as a PAD symptom when the exercise-pain-rest cycle was constant. Leg pain that occurred at rest was also diagnosed as PAD when the patient had experienced typical intermittent claudication. Complaints such as numbness, tingling and weakness of the lower-limb were not included in the PAD symptoms, because it was difficult to distinguish them from symptoms of peripheral neuropathy. Orthopedic diseases in the lumber supine were also excluded. Among the 102 diabetic patients, 9 showed diagnostic symptoms of PAD. The diagnosis was confirmed by angiography. Five

Table 1. Clinical characteristics of the subjects.

\begin{tabular}{lcccc}
\hline & \multirow{2}{*}{ Healthy subjects } & \multicolumn{2}{c}{ Type 2 diabetes } & \multirow{2}{*}{$P$ value } \\
\cline { 3 - 4 } & & PAD $(-)$ & PAD $(+)$ & \\
\hline Number of subjects & 101 & 93 & 9 & - \\
Gender (male/female) & $50 / 51$ & $47 / 46$ & $7 / 2$ & $0.939^{* *}$ \\
Age (years) & $57.6 \pm 7.9$ & $57.8 \pm 7.6$ & $67.4 \pm 7.1^{\mathrm{a}, \mathrm{b}}$ & 0.001 \\
Body mass index $\left(\mathrm{kg} / \mathrm{m}^{2}\right)$ & $22.6 \pm 3.0$ & $23.6 \pm 3.6$ & $24.8 \pm 6.3$ & 0.045 \\
Duration of diabetes (years) & - & $6(0-33)$ & $11(1-40)^{\mathrm{b}}$ & 0.212 \\
Systolic blood pressure (mmHg) & $122 \pm 15$ & $129 \pm 19^{\mathrm{a}}$ & $34 \pm 13$ & 0.008 \\
Diastolic blood pressure (mmHg) & $77 \pm 10$ & $78 \pm 10$ & $72 \pm$ & 0.130 \\
Diabetes therapy (LSM/OHA/NS) & - & $29 / 49 / 15$ & $2 / 7 / 0$ & $0.271^{* *}$ \\
Anti-hypertensive therapy (-//) & - & $62 / 31$ & $5 / 4$ & $0.503^{* *}$ \\
Anti-hyperlipidemic therapy (-/+) & - & $60 / 33$ & $6 / 3$ & $0.897^{* *}$ \\
Fasting plasma glucose (mmol/L) & $5.4 \pm 0.7$ & $9.4 \pm 3.5^{\mathrm{a}}$ & $7.8 \pm 2.4^{\mathrm{a}}$ & $<0.001$ \\
HbA1c (\%) & $5.0 \pm 0.4$ & $8.9 \pm 2.3^{\mathrm{a}}$ & $7.7 \pm 1.9^{\mathrm{a}}$ & $<0.001$ \\
Fasting plasma insulin (pmol/L) & $35(12-156)$ & $40(7-169)$ & $54(15-226)$ & $0.568^{*}$ \\
Total cholesterol (mmol/L) & $5.40 \pm 0.80$ & $5.43 \pm 1.06$ & $4.91 \pm 1.09$ & 0.295 \\
Triglyceride (mmol/L) & $1.14(0.61-18.7)$ & $1.26(0.43-4.97)$ & $1.33(0.67-1.83)$ & $0.589^{*}$ \\
HDL cholesterol (mmol/L) & $1.58 \pm 0.49$ & $1.40 \pm 0.41^{\mathrm{a}}$ & $1.34 \pm 0.31$ & 0.023 \\
\hline
\end{tabular}

Mean \pm SD or median (range).

$P$ values by analysis of variance, by Kruskal-Wallis test* or by chi-square test**.

${ }^{a} P<0.05$ vs. healthy control , ${ }^{b} P<0.05$ vs. DM-PAD (-) by Scheffe's F-test.

Abbreviations: PAD, peripheral artery disease; LSM, lifestyle modification; OHA, oral hypoglycemic agents; INS, insulin; HbA1c, hemoglobin A1c; HDL, high density lipoprotein. 
out of the 9 PAD patients underwent percutaneous transluminal angioplasty, and they were re-examined for changes in PWV and $\mathrm{ABI}$ after angioplasty.

The healthy subjects were participants of a local health check program in Osaka City. Exclusion criteria were presence of overt hyperglycemia of $7.0 \mathrm{mmol} / \mathrm{L}(126 \mathrm{mg} /$ $\mathrm{dL}$ ) or higher, liver dysfunction as defined by serum ALT $>50 \mathrm{IU}$, and overt proteinuria. In addition, we excluded those who were receiving medication for diabetes mellitus, dyslipidemia and/or hypertension.

As shown in Table 1, there was no significant difference between the healthy group and the diabetic subjects without PAD in age, gender and body mass index, whereas the diabetic patients with PAD were significantly older than the other two groups. In addition, the diabetic patients with PAD had a longer duration of diabetes than the non-PAD diabetic patients.

\section{Methods}

\section{Measurements of brachial-ankle PWV and ABI}

We measured brachial-ankle PWV (baPWV) and ABI simultaneously using an automatic device (11). Briefly, measurements were performed in the supine position after $5 \mathrm{~min}$ bed rest. Cuffs for occlusion and sensing were adapted to both arms and both ankles. Electrocardiogram was monitored with electrodes placed on both wrists. Heart sounds S1 and S2 were detected using a microphone set on the left edge of the sternum at the third intercostal space. Pulse pressure waveforms of brachial and tibial arteries were simultaneously recorded using an automatic waveform analyzer (model BP203RPE, Colin, Komaki, Japan) to measure the time interval between the initial rises of these waveforms (Tba in sec). The distance from the aortic orifice to the brachial sensor (Dhb) was calculated using the following equation (17); Dhb $(\mathrm{cm})=0.2195 \times \mathrm{HT}-2.0734(\mathrm{HT}$ : the height of the patient in $\mathrm{cm}$ ). Similarly, the distance from the aortic orifice to the ankle sensor (Dha) was represented as the length from aortic orifice to femur plus that from femur to the ankle, and calculated as follows; Dha $(\mathrm{cm})=(0.5643 \times \mathrm{HT}-18.381)+(0.2486 \times \mathrm{HT}+30.709)$. The waveform analyzer calculated baPWV using the following formula; baPWV $(\mathrm{cm} / \mathrm{sec})=($ Dha $-\mathrm{Dhb}) / \mathrm{Tba}$. Reproducibility of the baPWV measurement was evaluated by repeating measurements in 17 healthy subjects on 2 different occasions. The coefficient of variation (CV) was less than $5 \%$, which was comparable with the CV reported by Tomiyama et al. (17).

$\mathrm{ABI}$ was calculated as an ankle-to-brachial ratio of systolic blood pressure measured by an oscillometric method with cuffs adapted to extremities.

\section{Other measurements}

Blood was drawn in the morning after an overnight fast to measure glucose, insulin, total cholesterol, triglycerides, high-density lipoprotein (HDL) cholesterol, and $\mathrm{HbA1c}$. These assays were done by standard laboratory methods.

\section{Statistical analysis}

Valued are summarized as means \pm standard deviation (SD) for continuous variables with normal distribution. Comparison of mean values between groups was performed by one-way analysis of variance (ANOVA) with Scheffe's F-test. The effects of two independent factors on one dependent variable were evaluated by two-way ANOVA. Multiple regression analyses were performed to evaluate the independent effects of 3 or more clinical factors on one dependent variable. For continuous variables that showed a skewed distribution, values are summarized as median (range), and comparison between groups was done by non-parametric statistics (MannWhitney's U test or Kruskal-Wallis test). Wilcoxon test was used for comparison of paired data. Correlation of two parameters was assessed by Spearman's rank correlation method. $P$ values $<0.05$ were considered statistically significant. These statistical analyses were performed using statistics software (StatView version 5.0, SAS Institute, Cary, NC).

\section{Results}

\section{Association of age and diabetes with baPWV}

Since there was no significant difference in baPWV between the right and left legs in both the healthy subjects and the diabetic patients without PAD, we used the values of baPWV in the right legs in these groups for analysis. Among the subjects without PAD, age and presence of diabetes mellitus were independently associated with increased baPWV (Fig. 1).

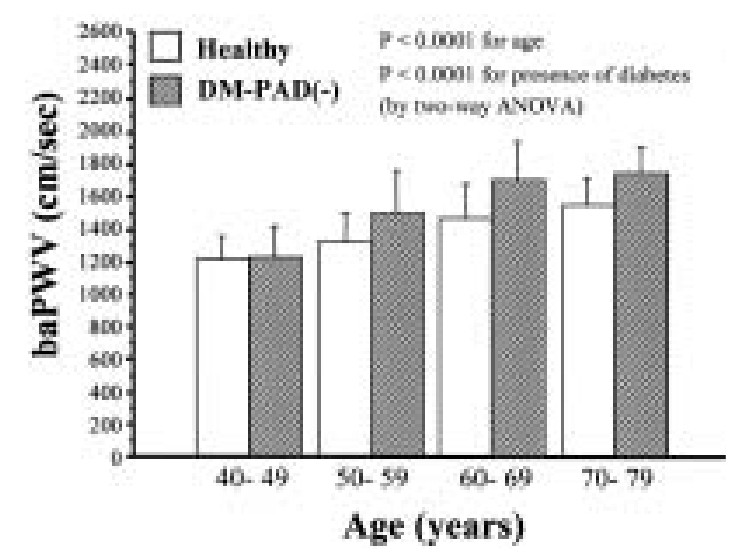

Fig. 1. Effects of age and presence of diabetes on baPWV in the healthy subjects and the diabetic patients without PAD Mean \pm SD.

$P$-values by two-way analysis of variance. 
Multiple regression analysis suggested that presence of diabetes (standard regression coefficient $\beta=0.241, P$ $<0.0001)$, age $(\beta=0.337, P<0.0001)$ and systolic blood pressure $(\beta=0.480, P<0.0001)$ were independently associated with increased baPWV (coefficient of determination $\left.\mathrm{R}^{2}=0.524, P<0.0001\right)$. Within the 93 diabetic patients without $\mathrm{PAD}$, age $(\beta=0.214, P=0.013)$, duration of diabetes $(\beta=0.167, P=0.034)$ and systolic blood pressure $(\beta=0.552, P<0.0001)$ were independently associated with baPWV $\left(\mathrm{R}^{2}=0.596, P<0.0001\right)$.

\section{Influence of PAD on baPWV}

Within the diabetic patients with PAD, the affected leg showed significantly lower baPWV than the non-affected legs (1221 (876-1910) vs. $1607(1251-1973) \mathrm{cm} / \mathrm{sec}, P=$ 0.021 by Wilcoxon test).

We compared $A B I$ and baPWV in the affected legs of diabetic patients with and without PAD with those of the non-PAD diabetic patients. We selected diabetic patients without PAD in the same age categories $(60$ s and 70 s, $N$ $=33$ ), because the PAD patients were significantly older than other groups. The PAD group showed significant reduction in both $A B I$ and baPWV compared with the non-PAD group (Fig. 2 A, B).
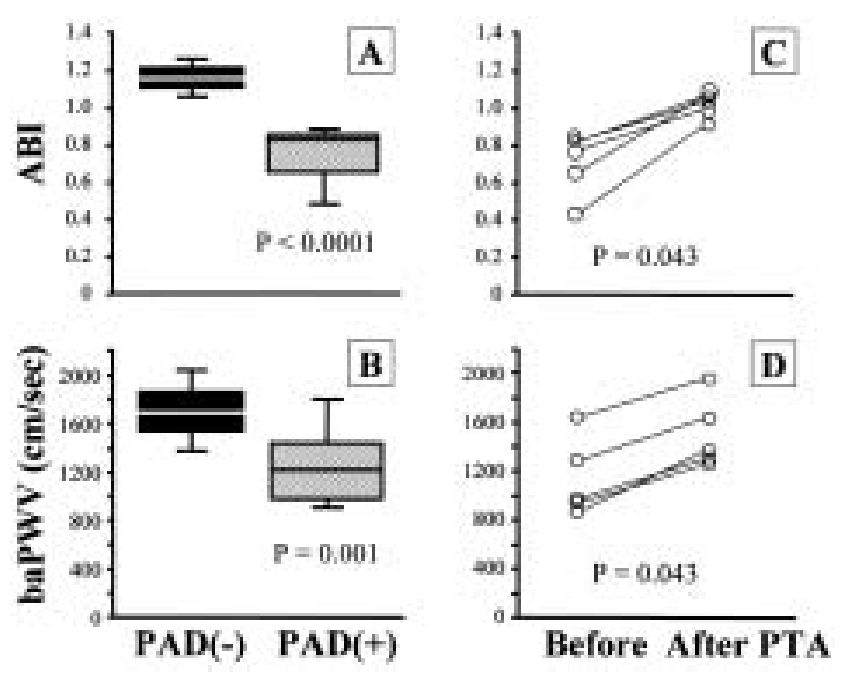

Fig. 2. Effect of $P A D$ on $A B I$ and baPWV.

Panels $A$ and $B$ : Comparison of $A B I$ (panel $A$ ) and baPWV (panel B) between 9 diabetic patients with PAD) and 93 diabetic patients without PAD in the same age categories (60s and 70s). Horizontal bars indicate the 10th, 25th, 50th, 75th, and 90th percentile levels. $P$ values are by Mann-Whitney's $U$ test.

Panels C and D: Increased ABI (panel C) and baPWV (panel D) in 5 PAD patients following successful percutaneous transluminal angioplasty. $\mathrm{P}$ values are by Wilcoxon test. $\mathrm{ABI}$, ankle-brachial pressure index.

$P A D$, peripheral arterial disease of the lower extremities. PTA, percutaneous transluminal angioplasty.
Five patients with PAD underwent percutaneous transluminal angioplasty. Following successful vessel dilatation, all of these patients showed a significant increase in not only $A B I$ but also in baPWV of the treated leg (Fig. 2 C, D).

The association between the presence of PAD and reduced baPWV was further analyzed by a multiple regression model. In the analysis of the 102 total diabetic patients, baPWV was inversely associated with the presence of PAD ( $\beta=-0.458, P<0.0001)$, whereas it was positively associated with age $(\beta=0.300, P<0.0002)$ and systolic blood pressure $(\beta=0.498, P<0.0001)$. This model explained $53.7 \%$ of the variation in baPWV.

\section{Association between PAD and the right-left difference in baPWV}

We calculated the absolute right-left difference in baPWV for each subject, and compared it among the three groups (Fig. 3). The medians of the absolute rightleft difference in baPWV were 36, 55, and $290 \mathrm{~cm} / \mathrm{sec}$ for the healthy subjects, the diabetic patients without $P A D$, and the diabetic patients with PAD, respectively. The difference among the groups was highly significant. In the diabetic patients with PAD, the absolute rightleft difference in baPWV showed a significant inverse correlation with $\mathrm{ABI}$ (Spearman's correlation coefficient $r_{\mathrm{s}}=0.783, P=0.027$, Fig. 4).

\section{Discussion}

In the present study, we measured baPWV in healthy subjects and type 2 diabetic patients with and without $P A D$. Although baPWV increased with age, systolic blood

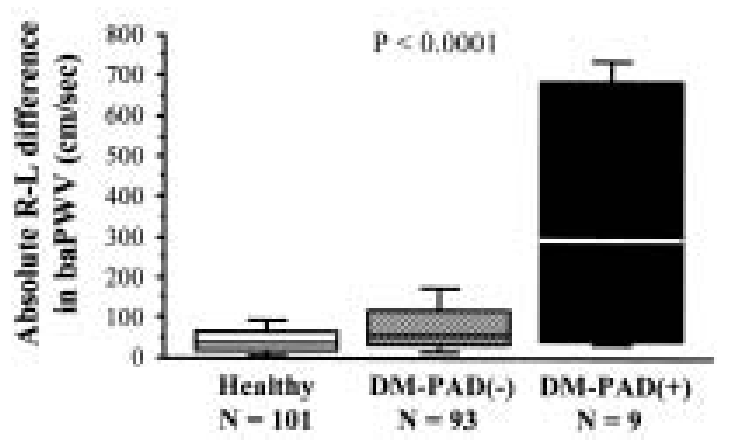

Fig. 3. Comparison of the absolute right-left difference in baPWV among the groups.

The median of the absolute right-left difference in baPWV was 36,55 , and $290 \mathrm{~cm} / \mathrm{sec}$ for the healthy subjects, diabetic patients without PAD, and diabetic patients with PAD, respectively.

Horizontal bars indicate the 10th, 25th, 50th, 75th, and 90th percentile levels.

$P$ value by Kruskal-Wallis test. 
pressure and presence of diabetes in subjects without $P A D$, patients with PAD had significantly lower baPWV than those without. Presence of PAD was an independent factor associated with lower baPWV among the patients with type 2 diabetes mellitus as shown by multiple regression analysis. Furthermore, the reduced baPWV showed a significant increase following successful dilatation of the affected arteries. These results clearly suggest that presence of PAD reduces baPWV.

We first expected that those with PAD had a higher baPWV, because those with advanced atherosclerosis have a higher aortic PWV. Although the opposite was the reality, the present results were consistent with previous findings by Simonson et al. (14) and Eliakim et al. (15) showing that PWV levels in the heart-feet and the femoral-dorsal segments were reduced in PAD. In contrast, Safar et al. (13) reported that brachial-radial PWV in patients with PAD was within the normal range. Taken together, patients with lower-limb PAD do not necessarily have changes in PWV of arteries in the upper extremities, whereas they have reduced PWV in the leg arteries. The reduction in baPWV would be attributable to arterial stenosis, because baPWV increased following successful dilatation of the stenosed arteries. As far as we know, this is the first documentation in the English literature that the reduced PWV in lower-limb arteries is reversible following successful treatment of the affected arteries. There are several explanations for the reduced baPWV in PAD. First, significant stenosis results in reduced blood pressure downstream that could suppress pulse wave propagation; PWV is dependent on blood pressure (18). In fact, both blood pressure at the ankle (or $A B I$ ) and baPWV were lower in those with PAD before angioplasty,

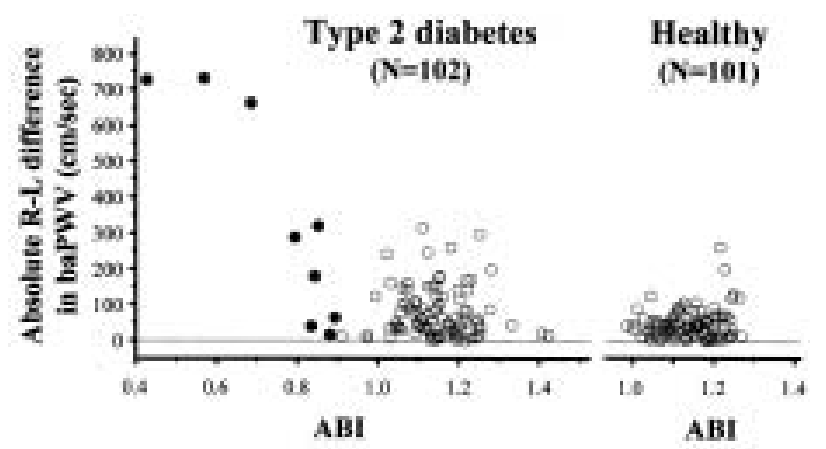

Fig. 4. Correlation between $\mathrm{ABI}$ and the absolute right-left difference in baPWV.

In the diabetic patients with PAD (closed circles), the absolute right-left difference in baPWV showed a significant inverse correlation with $\mathrm{ABI}$ (Spearman's correlation coefficient $r_{\mathrm{s}}=0.783$, $P=0.0267$ ). Such a relation was not significant in those without PAD (open circles) regardless of the presence of diabetes mellitus. and the increased baPWV after angioplasty was associated with increased $\mathrm{ABI}$. Second, it may take longer time for pulse waves to travel along collateral pathways as suggested in previous studies (15).

Sclerotic change in arteries increases baPWV, whereas presence of stenosis in the lower-limb arteries decreases it. Because of these combined effects, it is difficult to diagnose PAD based solely on baPWV. However, the difference in baPWV between the right and left legs may be of diagnostic value. As shown in this study, baPWV was significantly lower in the affected side than the other side. In addition, the absolute right-left difference in baPWV was significantly greater in those with lower ABI. Reduction of $A B I$ is widely used for screening lower-limb PAD (19). However, one of the limitations of this method is that PAD patients with arterial calcification show an increase, instead of decrease, in ABI (20). Combined measurements of $A B I$ and baPWV may increase the diagnostic power in screening PAD. Careful interpretation is needed in some cases, such as those with PAD in the bilateral legs and those with extremely high baPWV, because these patients would show falsely low and high right-left differences in baPWV, respectively.

In conclusion, the present study showed that baPWV was decreased in the affected legs in diabetic patients with PAD. In addition, the absolute right-left difference in baPWV was significantly greater in those with PAD. These results suggest that widening of the right-left difference in baPWV is useful for detecting PAD.

\section{References}

(1) Kannel WB and McGee DL: Update on some epidemiologic features of intermittent claudication: the Framingham Study. J Am Geriatr Soc, 33: 13-18, 1985

(2) Lehto S, Ronnemaa T, Pyorala K and Laakso M: Risk factors predicting lower extremity amputations in patients with NIDDM. Diabetes Care, 19: 607612, 1996

(3) Reunanen A, Takkunen $\mathrm{H}$ and Aromaa A: Prevalence of intermittent claudication and its effect on mortality. Acta Med Scand, 211: 249-256, 1982

( 4 ) O’Rourke M: Mechanical principles in arterial disease. Hypertension, 26: 2-9, 1995

( 5 ) Bouthier JD, De Luca N, Safar ME and Simon AC: Cardiac hypertrophy and arterial distensibility in essential hypertension. Am Heart J, 109: 1345-1352, 1985

(6) London GM, Marchais SJ, Safar ME, Genest AF, Guerin AP, Metivier F, Chedid K and London AM: Aortic and large artery compliance in end-stage renal failure. Kidney Int, 37: 137-142, 1990

( 7 ) Blacher J, Guerin AP, Pannier B, Marchais SJ, Safar ME and London GM: Impact of aortic stiffness on 
survival in end-stage renal disease. Circulation, 99: 2434-2439, 1999

( 8 ) Shoji T, Emoto M, Shinohara K, Kakiya R, Tsujimoto Y, Kishimoto H, Ishimura E, Tabata T and Nishizawa Y: Diabetes mellitus, aortic stiffness, and cardiovascular mortality in end-stage renal disease. J Am Soc Nephrol, 12: 2117-2124, 2001

( 9 ) Meaume S, Benetos A, Henry OF, Rudnichi A and Safar ME: Aortic pulse wave velocity predicts cardiovascular mortality in subjects \&gt;70 years of age. Arterioscler Thromb Vasc Biol, 21: 2046-2050, 2001

(10) Taniwaki H, Shoji T, Emoto M, Kawagishi T, Ishimura E, Inaba M, Okuno $Y$ and Nishizawa Y: Femoral artery wall thickness and stiffness in evaluation of peripheral vascular disease in type 2 diabetes mellitus. Atherosclerosis, 158: 207-214, 2001

(11) Suzuki E, Kashiwagi A, Nishio Y, Egawa K, Shimizu S, Maegawa H, Haneda M, Yasuda H, Morikawa S, Inubushi T and Kikkawa R: Increased arterial wall stiffness limits flow volume in the lower extremities in type 2 diabetic patients. Diabetes Care, 24: 21072114, 2001

(12) Haynes F, Ellis L and Weiss S: Pulse wave velocity and arterial elasticity in arterial hypertension, arteriosclerosis and related conditions. Am Heart J, 11: 385-401, 1936

(13) Safar ME, Laurent S, Asmar RE, Safavian A and London GM: Systolic hypertension in patients with arteriosclerosis obliterans of the lower limbs. Angiology, 38: 287-295, 1987

(14) Simonson E, Koff S, Keys A and Minckler J: Contour of the toe pulse, reactive hyperemia, and pulse transmission velocity: group and repeat variability, effect of age, exercise, and disease. Am Heart J, 50: 260-279, 1955

(15) Eliakim M, Sapoznikov D and Weinman J: Pulse wave velocity in healthy subjects and in patients with various disease states. Am Heart J, 82: 448457, 1971

(16) The expert committee on the diagnosis and classification of diabetes mellitus: Report of the expert committee on the diagnosis and classification of diabetes mellitus. Diabetes Care, 24: S5-S20, 2001

(17) Tomiyama H, Yamashina A, Arai T, Hirose K, Koji Y, Chikamori T, Hori S, Yamamoto Y, Doba N and Hinohara S: Influences of age and gender on results of noninvasive brachial-ankle pulse wave velocity measurement-a survey of 12517 subjects. Atherosclerosis, 166: 303-309, 2003

(18) Steele J: Interpretation of arterial elasticity from measurements of pulse wave velocities. 1. Effect of pressure. Am Heart J, 6: 452-465, 1937

(19) Orchard TJ and Strandness DE, Jr.: Assessment of peripheral vascular disease in diabetes. Report and recommendations of an international workshop sponsored by the American Diabetes Association and the American Heart Association September 1820, 1992 New Orleans, Louisiana. Circulation, 88: 819-828, 1993

(20) Brooks B, Dean R, Patel S, Wu B, Molyneaux L and Yue DK: TBI or not TBI: that is the question. Is it better to measure toe pressure than ankle pressure in diabetic patients? Diabet Med, 18: 528-532, 2001 